\title{
О СПОСОБЕ ВЫЧИСЛЕНИЯ ОТРАЖЕНИЯ АКУСТИЧЕСКОГО ИМПУЛЬСА ОТ СИСТЕМЫ ЖИДКИХ СЛОЕВ ПРИ НОРМАЛЬНОМ ПАДЕНИИ
}

N. VEKSLER. VEDELATE KIHTIDE SOSTEEMILE NORMAALI SIHIS LANGEVATE AKUSTILISTE IMPULSSIDE PEEGELDUSTE ARVUTAMISEST

N. VEKSLER. A METHOD FOR THE CALCULATION OF SOUND PULSE REFLECTION FROM A SYSTEM OF LIQUID LAYERS IN CASE OF NORMAL INCIDENCE

(Представлена Н. Алумяэ)

Рассмотрим систему из $n$ плоских жидких слоев, контактирующих с двумя жидкими полупространствами. Положим, что свойства слоев по толщине не изменяются.

Введем следующие обозначения: $x$ - декартова координата, отсчитываемая от границы верхнего полупространства в сторону жидких слоев, $t-$ время; для произвольного слоя $k$ назовем: $p_{k}-$ акустическое давление, $U_{k}$ - компонента вектора перемещения, $\varrho_{k}-$ плотность, $c_{k}$ - скорость звука, $\zeta_{k}=\varrho_{k} c_{k}$ - акустический импеданс, $h_{k-1}\left(h_{k}\right)$ расстояние от границы $x=0$ до лицевой (тыльной) поверхности слоя $k$, $l_{k}=h_{k}-h_{k-1}-$ толщина слоя, $t_{k}=l_{k} / c_{k} \ldots$ время пробега акустического импульса по слою. Те же величины с индексом нуль будем относить к падающему импульсу, без индекса - к отраженному импульсу в верхнем полупространстве, с индексом $n+1-$ к нижнему полупространству.

Положим, что время $t$ отсчитывается с момента, когда падающий импульс достиг лицевсй поверхности $x=0$. До этого времени все жидкие слои и граничащие с ними жидкие полупространства находились в покое и имели место нулевые начальные условия

$p_{0}=p=p_{k}=0, \quad \partial p_{0} / \partial t=\partial p / \partial t=\partial p_{k} / \partial t=0 \quad$ при $\quad t=0$

$U_{0}=U=U_{k}=0, \quad \partial U_{0} / \partial t=\partial U / \partial t=\partial U_{k} / \partial t=0 \quad(k=1,2,3, \ldots, n+1)$.

Пусть из верхнего жидкого полупространства на границу $x=0$ нормально к ней падает плоский акустический импульс давления с произвольным законом изменения интенсивности

$$
p_{0}=f(t-x / c) H(t-x / c), \quad H(t-x / c)=\left\{\begin{array}{l}
1, t-x / c>0 \\
0, t-x / c \leqslant 0
\end{array}\right.
$$

где $f-$ заданная функция.

Полагаем, что каждый из слоев и жидкие полупространства описываются одномерными волновыми уравнениями относительно соответствующего акустического давления 
$\left(\partial^{2} / \partial x^{2}-c^{-2} \partial^{2} / \partial t^{2}\right) p=0$

$x \leqslant 0$,

$\left(\partial^{2} / \partial x^{2}-c_{k}^{-2} \partial^{2} / \partial t^{2}\right) p_{k}=0 \quad(k=1,2,3, \ldots, n)$,

$h_{k} \geqslant x \geqslant h_{k-1}$,

$\left(\partial^{2} / \partial x^{2}-c_{n+1}^{-2} \partial^{2} / \partial t^{2}\right) p_{n}=0$

$x \geqslant h_{n}$.

С учетом нулевых начальных условий (1) представим условия контакта слоев

$$
\begin{aligned}
& p_{0}+p=p_{1}, \quad O_{0}+U=U_{1}, \quad x=0, \\
& p_{k}=p_{k+1}, \quad U_{k}=U_{k+1} \quad(k=1,2,3, \ldots, n), \quad x=h_{k} .
\end{aligned}
$$

Считаем, что в нижнем полупространстве от границы $x=h_{n}$ распространяется только импульс в положительном направлении координаты $x$.

Применив к сформулированной задаче по времени $t$ интегральное преобразование Лапласа, выпишем решение изображающей задачи

$$
\begin{array}{ll}
p^{L}=A \exp \left(c^{-1} x s\right), & x \leqslant 0, \\
p_{k}^{L}=A_{k} \exp \left(c_{k}^{-1} x s\right)+B_{k}\left(-c_{k}^{-1} x s\right), & h_{k} \geqslant x \geqslant h_{k-1}, \\
p_{n+1}^{L}=B_{n+1} \exp \left(-c_{n+1}^{-1} x s\right), & x \geqslant h_{n} .
\end{array}
$$

Величины $A_{k}, B_{k}$ находятся из алгебраической системы уравнений $A+a_{12} A_{1}=a_{1}, \quad a_{22} A_{1}+B_{1}=a_{2}$,

$\dot{a}_{2 k+1,2 k} \dot{A}_{k}+\dot{a}_{2 k+1,2 k+1} B_{k}+a_{2 k+1,2 k+2} A_{k+1}=\dot{0}$,

$a_{2 k+2,2 k+1} B_{k}+a_{2 k+2,2 k+2} A_{k+1}+a_{2 k+2,2 k+3} B_{k+1}=0$,

$\dot{a}_{2 n+1,2 n} \dot{A}_{n} \dot{+} a_{2 n+1,2 n+1} \dot{B}_{n}=\dot{0}$,

$a_{2 n+2,2 n+1} B_{n}+a_{2 n+2,2 n+2} B_{n+1}=0 \quad(k=1,2,3, \ldots, n)$.

Коэффициенты этой системы уравнений имеют следующие значения: $a_{12}=-u, a_{22}=v, a_{1}=v f^{L}(s), a_{2}=w f^{L}(s), a_{2 k+1,2 k}=-\alpha_{k, k}$, $a_{2 k+1,2 k+1}=\beta_{k, k} v_{k}, a_{2 k+1,2 k+2}=\alpha_{k, k+1} u_{k}, a_{2 k+2,2 k+1}=-\beta_{k, k} w_{k}$, $a_{2 k+2,2 k+2}=\alpha_{k, k+1} v_{k}, a_{2 k+1,2 k+3}=\beta_{k, k+1}(k=1,2,3, \ldots, n)$, $a_{2 n+1,2 n}=-\alpha_{n, n}, a_{2 n+1,2 n+1}=\beta_{n, n} v_{n}, a_{2 n+2,2 n+1}=-\beta_{n, n} w_{n}$,

$a_{2 n+1,2 n+2}=\beta_{n, n+1}, \alpha_{k, k+1}=\exp \left(c_{k+1}^{-1} h_{k} s\right), \beta_{k, k+1}=\exp \left(-c_{k+1}^{-1} h_{k} s\right)$,

$u_{k}=2 \xi_{k} /\left(1+\xi_{k}\right), v_{k}=\left(1-\xi_{k}\right) /\left(1+\xi_{k}\right), w_{k}=2 /\left(1+\xi_{k}\right)$,

$u_{k} w_{k}+v_{k}^{2}=1 \quad(k=1,2,3, \ldots, n), \xi=\zeta / \zeta_{1}, \xi_{k}=\zeta_{k} / \zeta_{k+1}$.

Как известно, в формулах Френеля величины $u_{k}, v_{k}, w_{k}$ называются, соответственно, коэффициентами прохождения из слоя $k+1$ в слой $k$, отражения от слоя $k$ в слой $k-1$, прохождения из слоя $k-1$ в слой $k$. Коэффициенты системы уравнений (7) находятся рекуррентно. В случае $n$ жидких слоев приходится решать систему из $2(n+1)$ алгебраических уравнений. В записи (7) матрица коэффициентов при неизвестных этой системы - ленточная и состоит из главной диагонали и двух соседних с ней. Из свободных коэффициентов ненулевых - только два. Такая форма системы уравнений значительно облегчает ее решение. 
В качестве примера приведем решение такой системы уравшений для случая трех жндких слоев. Для коэффициента $A$ (5) получим

$$
\begin{array}{r}
A=f^{L}(s)\left[v+\delta_{1}\left(1-v^{2}\right) \sigma_{1} \sigma_{2}^{-1}\right], \quad \sigma_{1}=v_{1}+\delta_{2} v_{2}+\delta_{3} v_{1} v_{2} v_{3}+\delta_{2} \delta_{3} v_{3}, \\
\sigma_{2}=1+\varepsilon, \quad \varepsilon=\delta_{1} v \sigma_{1}+\delta_{2} v_{1} v_{2}+\delta_{3} v_{2} v_{3}+\delta_{2} \delta_{3} v_{1} v_{3} . \\
\delta_{k}=\exp \left(-2 s t_{k}\right) \\
(k=1,2,3) .
\end{array}
$$

Характерной особенностью последней формулы является то обстоятельство, что в нее, помимо $f^{L}(s)$, входят коэффициенты отражения $v_{k}$ и изображения смещений по времени $\delta_{k}$.

Поскольку $|\varepsilon|<1$, используем разложение $\sigma_{2}^{-1}=\sum_{m=0}^{\infty}(-\varepsilon)^{m}$ и представим величину $A$ в виде ряда

$$
A=f^{L}(s)\left[v+\delta_{1}\left(1-v^{2}\right) \sigma_{1} \sum_{m=0}^{\infty}(-\varepsilon)^{m}\right] .
$$

Проведя в последней формуле перемножение и сгруппировав члены с одинаковыми степенями пронзведений $\delta_{k}$, после умножения на $\exp \left(c^{-1} x s\right) \quad(5)$ и использования теоремы смещения найдем оригинал акустического давления $p$ в отраженном от лицевой поверхности импульсе. В качестве примера приведем обращение одного характерного слагаемого формулы (9), стоящего при произведенин $\delta_{1} \delta_{2} \delta_{3}$ :

$$
y^{L}=f^{L} \exp \left(c^{-1} x S\right) \delta_{1} \delta_{2} \delta_{3}\left(1-v^{2}\right)\left(1-v_{1}^{2}-v_{2}^{2}+v_{1}^{2} v_{2}^{2}\right) v_{3},
$$

которое с помощью соотношения $1-v_{k}^{2}=u_{k} w_{k}(7)$ представим в виде

$$
y^{L}=F \exp \left(c^{-1} x s\right) \delta_{1} \delta_{2} \delta_{3} f^{L}(s), \quad F=u w u_{1} w_{1} u_{2} w_{2} v_{3} .
$$

Оригинал этого слагаемого запишем в форме

$$
y=F f(\tau) H(\tau), \quad \tau=t+c^{-1} x-2\left(t_{1}+t_{2}+t_{3}\right) .
$$

Такое слагаемое учитывает вклад в отраженный импульс в верхнем жидком полупространстве импульса, прошедшего туда и обратно все три жидких слоя. Это видно из его запаздывания на время $2\left(t_{1}+t_{2}+t_{3}\right)$ относительно импульса, сразу отразившегося от гранищы $x=0$, и интенсивности $F$, представляющей собой произведение коэффициентов прохождения $u_{k}$ из слоя $k+1$ в слой $k$, коэффициентов прохождения из слоя $k$ в слой $k+1$ и коэффициента отражения $v_{3}$ от нижнего жидкого полупространства к третьему жидкому слою.

Отметим, что импульсы, совершившие одинаковое число переотражений (в различной временной последовательности), согласно формуле (9) суммируются автоматически.

Полученные результаты могут найти применение при разработке автоматических акустических уровнемеров, используемых в различных технологических химических процессах и для контроля заполнения резервуаров-хранилищ жидкостей.

Как известно, при нормальном падении акустического импульса на систему упругих слоев (либо на систему упругих слоев, чередующихся с жидкими) поперечных волн в последних не возникает, так что в этом случае они ведут себя как жидкие. Поэтому полученные результаты могут служить основой для определения толщины и импеданса отдельного упругого слоя, расположенного в пакете слоев. 